\title{
A Multi-Radar Wireless System for Respiratory Gating and Accurate Tumor Tracking in Lung Cancer Radiotherapy
}

\author{
Changzhan Gu, Student Member, IEEE, Ruijiang Li, Steve B Jiang, and Changzhi Li, Member, IEEE
}

\begin{abstract}
Respiratory gating and tumor tracking are two promising motion-adaptive lung cancer treatments, minimizing incidence and severity of normal tissues and precisely delivering radiation dose to the tumor. Accurate respiration measurement is important in respiratory-gated radiotherapy. Conventional gating techniques are either invasive to the body or bring insufficient accuracy and discomfort to the patients. In this paper, we present an accurate noncontact means of measuring respiration for the use in gated lung cancer radiotherapy. We also present an accurate tumor tracking technique for dynamical beam tracking radiotherapy. Two $2.4 \mathrm{GHz}$ miniature radars were used to monitor the chest wall and abdominal movements simultaneously to get high resolution and enhanced parameter identification. Ray tracing technique was used to investigate the impact of antenna size in clinical practice. It is shown that our multiple radar system can reliably measure respiration signals for respiratory gating and accurate tumor tracking in motion-adaptive lung cancer radiotherapy.
\end{abstract}

\section{INTRODUCTION}

L UNG cancer is the number one cancer-related cause of death in both men and women through the world. It accounts for $15 \%$ of all cancer diagnoses and $28 \%$ of all cancer deaths. Lung cancer radiotherapy is subject to tumor motion which is mainly induced by respiration.

The respiration-induced organ motion greatly degrades the conformal radiotherapy by directing the radiation dose to the surrounding healthy tissues, which not only reduces the sufficient dose for the tumor, but also may cause severe side effects such as secondary cancer [1]. Respiratory gating and tumor tracking are two promising motion-adaptive techniques that allow free tumor motion but maintain a constant target position in the beam's eye view when the beam is on [2]. Respiratory gating limits radiation exposure to part of the respiration cycle when the tumor moves into the proper path window of the beam [1] [2]. There are mainly two kinds of gating techniques: internal gating and external gating. Internal gating is an invasive way that implants fiducial markers into the body. For lung cancer patients, it may bring serious risks such as pneumothroax. External gating, which uses external surrogates placed on the chest or abdomen, suffers from the lack of accuracy and brings discomfort to the

Manuscript received March 26, 2011.

Changzhan $\mathrm{Gu}$ and Changzhi Li are with the Department of Electrical and Computer Engineering, Texas Tech University, Lubbock, TX 79409, USA. (changzhan.gu@ttu.edu, changzhi.li@ttu.edu).

Ruijiang Li is with the Department of Radiation Oncology, Stanford University, Standford, CA 94305, USA. (rli2@stanford.edu).

Steve B Jiang is with the Department of Radiation Oncology, University of California, San Diego, CA 92307 USA (sbjiang@ucsd.edu). patients. Tumor tracking is the basis for the beam tracking technique that directs the radiation dose to the tumor dynamically by following the location of the tumor in real time. The tumor location information could be derived from respiration due to the internal organ link. However, beam tracking technique is still under research [1].

Doppler radar for measuring vital signs has been a hot research topic in recent years [3] [4] [5]. The noncontact radar method requires neither internal nor external surrogates, but provides reliable respiration measurements. Instead of measuring surrogate markers, the radar directly measures the chest wall and abdomen movement, which is more closely related to tumor motion. Moreover, microwave penetration makes the radar approach a more robust way of measuring accurate respiration signals.

In this paper, we used two miniature $2.4 \mathrm{GHz}$ Doppler radars to experimentally measure chest wall and abdomen respiration motions in a noncontact manner. The compact size allows integration of the radar to the linear accelerator (linac). The two movements were measured and wirelessly transmitted via a ZigBee module integrated in the radar circuit to another ZigBee receiver connected to a laptop. The received signals are then processed to generate gating signals for gated radiotherapy. We derived and compared the tumor tracking in two scenarios: respiration measured by external surrogates and by Doppler radar. We also analyzed the impact of antenna size on measurement accuracy in clinical practice. This radar method allows reliable measurement of respiration motions and accurate tracking of mobile tumors.

A multi-radar wireless radiotherapy system is introduced in Section II. In Section III, we analyze the measured signals and present the approach to generate gating signals. Section IV investigates the impact of antenna and potential interferences. In Section $\mathrm{V}$, we discuss the feasibility and reliability of using radar for accurate tumor tracking. A conclusion is made in Section VI.

\section{DOPPLER RADAR RADIOTHERAPY SYSTEM}

The setup of the multi-radar radiotherapy system is illustrated in Fig. 1. Multiple radars operating simultaneously form a MIMO system with increased virtual aperture identification [6]. In our experiment, two radars are hanged over the patient to monitor the real-time respiration movements of chest and abdomen respectively. The inset (a) shows the configuration of a $2 \times 2$ antenna array with axes, and inset (b) illustrates the respiratory-gated radiotherapy mechanism. The linear accelerator (linac) finds the tumor 


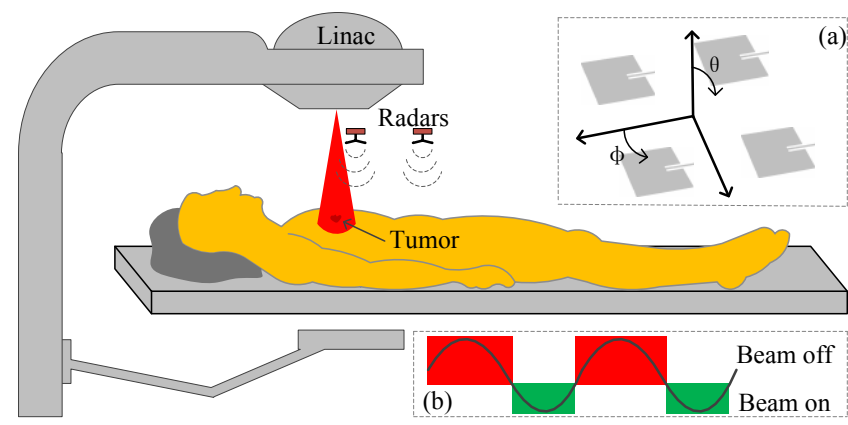

Fig.1. Setup of the multi-radar wireless radiotherapy system. Two radars are used to monitor the chest and abdomen respectively. Insets: (a) Configuration of a $2 \times 2$ antenna array with axes, and (b) mechanism of respiratory-gated radiotherapy.

location based on the geometry data obtained from the simulation/planning stage and delivers radiation dose with a certain beam width covering tumor and some safety margin [1]. Due to the internal organ link, the tumor moves around in response to the respiration movement. It is very likely that tumor may move out of the radiation coverage so that more healthy tissues will be radiated. In respiratory-gated radiotherapy, the linac turns on the radiation only when the tumor moves into the beam coverage so as to deliver the highest dose to the tumor while minimizing damage to healthy tissues. In beam tracking radiotherapy, the radiation follows the moving tumor dynamically based on the location data derived from real-time respiration signals. Fig. 2 shows the miniature $2.4 \mathrm{GHz}$ Doppler radar with ZigBee wireless transmission capability, making it easy to be integrated into the linac and released from a bundle of wires for data transmission. The inset shows the circuit block diagram of the designed $2.4 \mathrm{GHz}$ radar sensor. The radar was designed with homodyne architecture, which transmits a power of $0 \mathrm{dBm}$ that is almost one thousand times less than the peak power of an ordinary GSM cell phone. One ZigBee module is integrated in the radar circuit as a transmitting node and another module is connected to the laptop as a receiving node. Two patch antennas were designed in our experiments to collect data.

The Doppler radar radiates a single tone signal to the chest or abdomen. The periodic respiration movement modulates the single tone signal. By receiving and down-converting the reflected signal, the radar baseband $I / Q$ channel outputs are:

$$
\begin{aligned}
& B(t)_{I}=A_{I} \cos [\theta+4 \pi x(t) / \lambda+\Delta \phi(t)]+D C_{I} \\
& B(t)_{Q}=A_{Q} \sin [\theta+4 \pi x(t) / \lambda+\Delta \phi(t)]+D C_{Q}
\end{aligned}
$$

where $\theta$ is a constant phase offset determined by the initial position of the patient, $x(t)$ is the physiological motion caused by breathing, $\Delta \phi(\mathrm{t})$ is the residual phase noise, $A_{I} / A_{Q}$ are the amplitudes and $D C_{I} / D C_{Q}$ are the $\mathrm{DC}$ offsets of the $I / Q$ channels respectively. After careful calibration of amplitudes and DC offsets, arctangent demodulation is then utilized to accurately demodulate the phase-modulated signal [7].

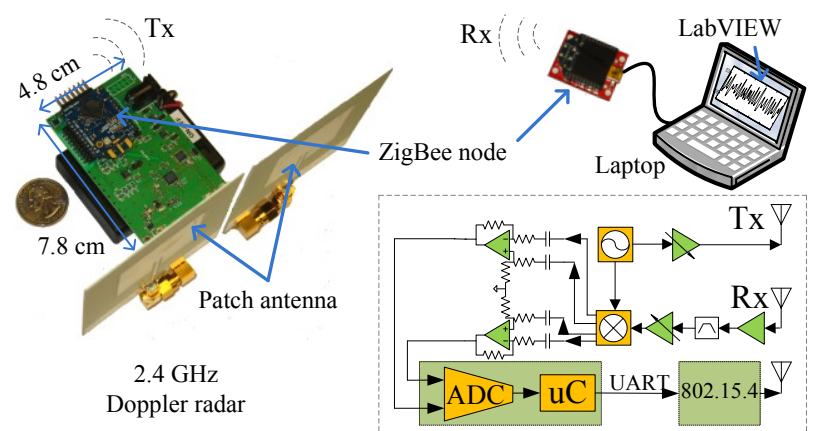

Fig.2. A $2.4 \mathrm{GHz}$ miniature Doppler radar $(7.8 \mathrm{~cm} \times 4.8 \mathrm{~cm})$. Inset shows the circuit block diagram. A ZigBee module is integrated in the radar circuit as the transmitting node and another module as the receiving node is connected to the laptop with LabVIEW running for signal processing.

(a)

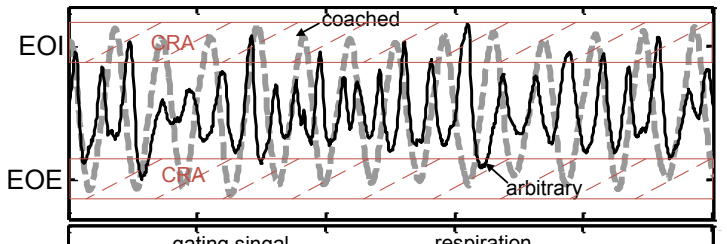

(b)

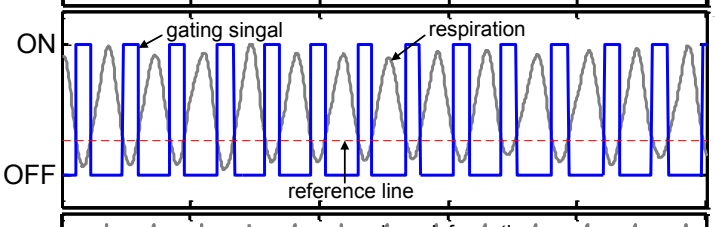

(c)

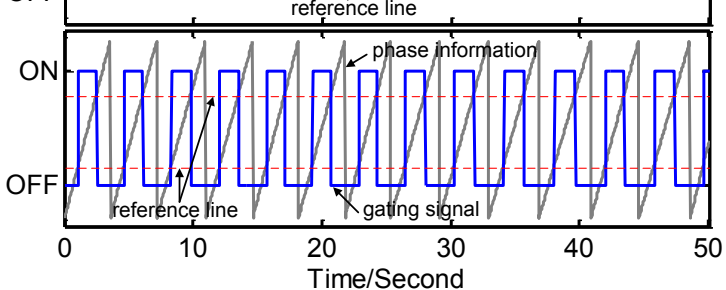

Fig. 3. (a) Measured respiration motion with an arbitrary rhythm and a coached rhythm. CRA: coaching reference area. (b) Amplitude gating with a duty cycle of $30 \%$. (c) Phase gating with a duty cycle of $40 \%$. The reference lines correspond to the threshold used to generate gating signals.

\section{RESPIRATORY GATING EXPERIMENT AND ANALYSIS}

Different people have different breathing patterns. They differ not only in rhythm but also in shape. In order to simulate the clinical practice, we carried out two sets of experiments using the $2.4 \mathrm{GHz}$ radar set: free breathing and coached breathing. In the experiments, the target person was seated about half a meter away from the radar radiating at the chest or abdomen. The measured respiration signals were wirelessly transmitted via the radar ZigBee node to another ZigBee node connected to the laptop with a real-time LabVIEW program running for signal processing.

In free breathing, the patient breathes naturally at his/her own arbitrary rhythm. On the other hand, coached breathing advises the patient to breathe by following some instructions that guide the breathing in real-time to a predefined pattern. The experimental results are shown in Fig. 3 (a). The shaded regions represent coaching reference of end-of-inspiration (EOI) and end-of-expiration (EOE). It is seen that arbitrary breathing may make it hard for respiratory gating. In the case of coached breathing, the target person is asked to look at the real-time visual feedback of respiration motions, obtained 


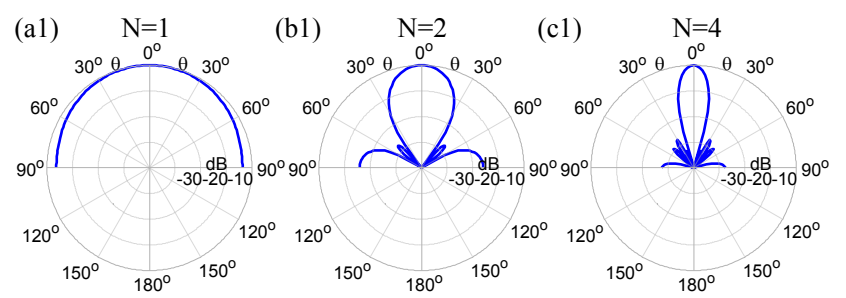

(a2)

(b2)

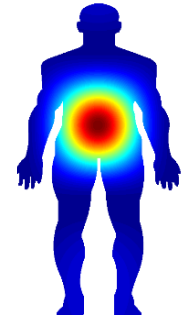

(a3)
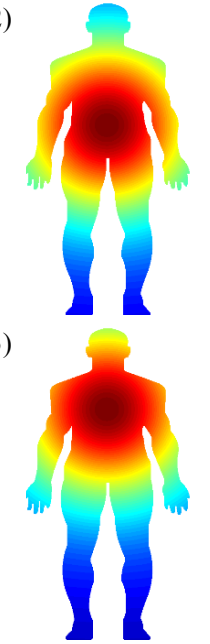

b3)

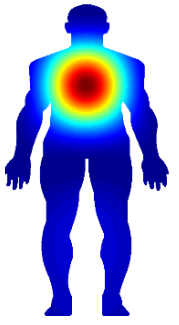

(c2)

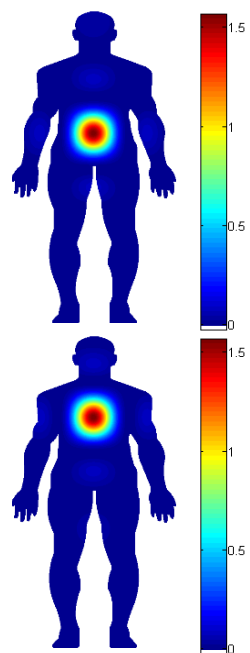

Fig. 4. Radiation pattern and intensity with different patch antenna arrays of (a1) $N=1$, (b1) $N=2$, and (c1) $N=4$, at abdomen: (a2), (b2), and (c2), and chest: (a3), (b3), and (c3).

from radar, from a LabVIEW window and adjust his breathing dynamically to put the EOI/EOE position within the coaching reference area [3]. Thus, more producible respiration signals are available, as shown in Fig.3 (a). The breathing coaching is important in lung-cancer radiotherapy not only because of the more reproducible respiration signals, but also because some lung-cancer patients do not have a regular breathing pattern, or cannot generate a reproducible breathing pattern. These patients should be identified in the simulation/planning stage and excluded from respiratory-gated radiotherapy.

The measured reproducible respiration signals allow both amplitude gating and phase gating. In amplitude gating, a reference line as shown in Fig. 3 (b) is included as the amplitude reference. The respiration signals trigger the beam on once its amplitude crosses below the reference line. Phase gating depends on the phase information derived from the respiration signals. Fig. 3 (c) illustrates a $40 \%$ phase gating, where the gray line represents the phase information ranging from $-\pi$ to $+\pi$, and the blue rectangular traces represent the corresponding gating signals for the linac to turn on/off. Whether to use amplitude gating or phase gating depends on the specific clinical case. The radar-measured reproducible respiration signals also allow adjustment of duty cycles to determine the best tradeoff between duty cycle and residual motion for each specific patient.

\section{ANTENNA AND INTERFERENCE ANALYSIS}

Antenna plays an important role in radio frequency (RF) (a)

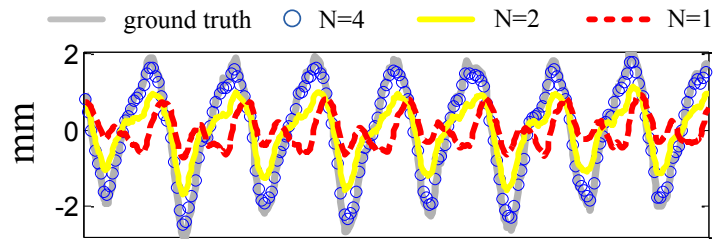

(b)

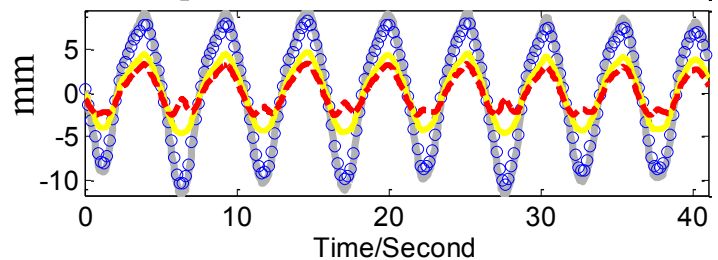

Fig. 5. Radar respiration measurement with different size of antenna arrays at: (a) chest, and (b) abdomen. Measured result approaches the ground truth with increasing antenna array size.

transceivers. The omnidirectional radiation pattern of a dipole or monopole antenna makes it susceptible to environmental interferences, such as multipath reflection, space electromagnetic waves, and so on. Patch antenna carries its merit of low-profile and high directivity. We designed a customized $2.4 \mathrm{GHz}$ patch antenna array for respiration measurement. An antenna array consisting of multiple single patch antennas results in higher gain and higher directivity. It not only helps deliver more RF power to the target so as to increase the sensitivity of the radar, but also helps prevent from environmental RF interference owing to its high directivity. However, in clinical practice, the available space in the linac may not allow us to use a big antenna array. Moreover, in our multi-radar radiotherapy system, two radars are used to simultaneously monitor the respiration motions of chest and abdomen. Since there is some phase offset between the chest motion and abdomen motion, accurate measurement requires that these two motions do not interfere with each other. However, due to the antenna beamwidth and the vicinity of chest and abdomen, it is unavoidable that chest motion and abdomen motion may be coupled to each other.

Therefore, it is necessary to discuss the antenna issue in radiotherapy application and investigate its impact on respiration measurement. Based on the patch antenna we designed, we built antenna arrays and carried out respiration measurement simulations using the ray tracking technique [8]. An $N \times N$ antenna array is assumed to be a two dimensional structure, of which $N$ is the number of single antenna elements along each dimension. Inset (a) in Fig. 1 shows a 2 $\times 2$ array with axes. Fig. 4 shows the radiation patterns of different antenna arrays of $N=1, N=2$ and $N=4$ along $\mathrm{Phi}=90^{\circ}$ plane. It is shown that the radiation pattern narrows down as the array size increases. Fig.4 also illustrates the radiation intensities at chest and abdomen respectively by using different size of antenna arrays. The simulation results of respiration measurement are illustrated in Fig.5. It is seen that the measured respiration signal approaches the ground truth reference signal as the antenna array size increases. This can be explained by the radiation intensity distribution as shown in Fig.4. As the antenna array size increases, the radiated area 


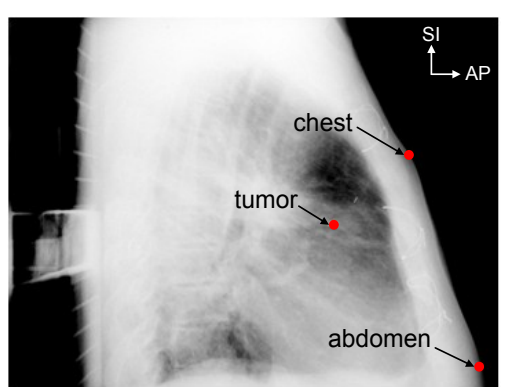

Fig. 6. An x-ray image with chest, abdomen, and tumor center marked. The motion trajectories of the three different objects were manually tracked by a clinician in each $x$-ray image.

on the body tends to decrease, so that less overlap occurs between the chest and abdomen radiation areas. Therefore, less chest motion would bother abdomen motion, and vice versa. Our experiments showed that a $2 \times 2$ antenna array is enough for accurate tumor tracking.

\section{ACCURATE TUMOR TRACKING}

Lung tumor motion is induced by respiratory motion. Respiration is a complex physiological process which results from the action of several muscles, primarily the intercostals muscles and the diaphragm. The motions of these internal anatomies are in turn closely related to the external chest wall motion and abdominal surface motion, which can be monitored by the radar system. Since the two motions are independent and somewhat different, in order to accurately determine the internal lung tumor motion, they have to be measured separately: each by a radar sensor.

To validate the overall tracking performance based on the radar measurements, we compared tumor motion obtained from simulated radar output with that measured by x-ray imaging. We took a series of 41-second long fluoroscopic $\mathrm{x}$-ray images from a lung cancer patient treated at a clinic. A clinician manually tracked the chest and abdomen motion along the anterior-posterior (AP) direction. In addition, the corresponding tumor motion along the superior-inferior (SI) direction was also tracked by the clinician, since the largest motion in lung mostly occurs in the SI direction. Fig. 6 shows a schematic plot of this procedure. The clinician-tracked results were used as the ground truth motion in this study. In this work, we used the artificial neural network to build the tracking algorithm. The particular network architecture we used is the feed-forward multiplayer perceptron (MLP) with one hidden layer. This type of neural network is a universal function approximator in the sense that if the number of neurons in the hidden layer can be made arbitrary large, then given sufficient training data, an MLP can approximate arbitrary relation between the input and output. Once the algorithm converges, the network weights are fixed and applied to new test data for validation purposes.

The first $\sim 13$ seconds were used as training data and the remaining $\sim 28$ seconds were used as test data. The training of the MLP converges after 10 epochs. The tracking algorithm is able to track both regular and irregular breathing patterns. Fig. (a)

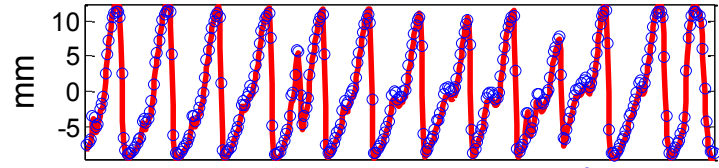

(b)

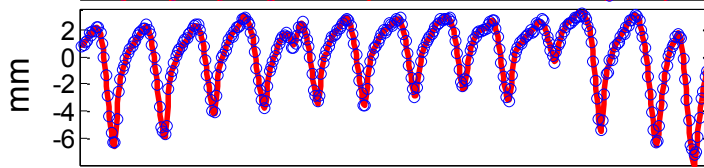

(c)

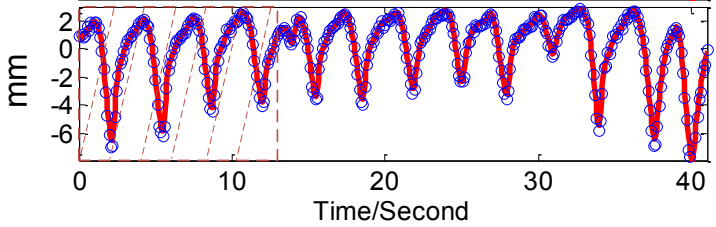

Fig. 7. Radar simulation (circle) and ground truth (solid line) for an irregular breathing pattern: (a) abdomen, (b) chest, (c) tumor tracking results. The shaded area separates the training and test datasets.

7 shows the tracking results of an irregular breathing pattern, which is a common case in clinical practice. The absolute tracking error is about $0.12 \mathrm{~mm}$. The absolute error at 91 percentile is about $0.33 \mathrm{~mm}$. This error is roughly on the same order with the actual radar measurement error and is sufficient for accurate beam tracking in lung cancer radiotherapy.

\section{CONCLUSION}

This paper presented a multi-radar wireless system for the use of respiratory gating and accurate tumor tracking in lung cancer radiotherapy. Analysis has been carried out to investigate the impact of antenna size in clinical practice. Experiments and analysis proved the reliability of using radar to generate respiratory gating signals. Respiratory motions measured from chest and abdomen are combined to obtain the tumor motion. It has been proved that the multi-radar system provides a reliable way for respiratory gating and accurate tumor tracking in lung cancer radiotherapy.

\section{REFERENCES}

[1] S.B. Jiang, "Technical aspects of image-guided respiration-gated radiation therapy," Medical Dosimetry, Vol.31(2), pp.141-151, 2006.

[2] S.B. Jiang, "Radiotherapy of mobile tumors," Seminars in Radiation Oncology, Vol. 16, Issue. 4, pp.239-24, October 2006.

[3] C. Gu, R. Li, C. Li, and S.B. Jiang, "Doppler Radar Respiration Measurement for Gated Lung Cancer Radiotherapy," IEEE Radio and Wireless Week, Phoenix AZ, 2011

[4] C. Li, X. Yu, C. Lee, D. Li, L. Ran, J. Lin, "High-sensitivity software-configurable 5.8-GHz radar sensor receiver chip in 0.13- um CMOS for noncontact vital sign detection," IEEE Transactions on Microwave Theory and Techniques, Vol. 58, No. 5, Part: 2, 2010.

[5] C. Gu, C. Li, J. Lin, J. Long, J. Huangfu, L. Ran, "Instrument-based noncontact Doppler radar vital sign detection system using heterodyne digital quadrature demodulation architecture," IEEE Transactions on Instrumentation and Measurement, Vol. 59, No. 6, pp.1580-88, 2010.

[6] J. Li, P. Stoica, and X. Zheng, "Signal Synthesis and Receiver Design for MIMO Radar Imaging." IEEE Transactions on Signal Processing, Vol. 56, No.8, pp. 3959-3968, 2008.

[7] S. Kim, C. Nguyen, "A displacement measurement technique using millimeter-wave interferometry," IEEE Transactions on Microwave Theory and Techniques, Vol.51 , No.6, pp: 1724-1728, 2003.

[8] C. Li, J. Lin, "Random Body Movement Cancellation in Doppler Radar Vital Sign Detection," IEEE Transactions on Microwave Theory and Techniques, vol. 56, issue 12, pp. 3143-3152, December 2008. 